\title{
Research on the Quantization Error Introduced by IEC61850-9-1/2 in Digital Electric Power Metering System
}

\author{
Yi Tang ${ }^{1,2,3, a}$, Binji Wang ${ }^{1,2}$, Bo Jiang ${ }^{2}$ \\ ${ }^{1}$ State Grid Sichuan Electric Power Corporation Electrical Science Research Institute, Chengdu, \\ 610018, China, \\ ${ }^{2}$ State Grid Sichuan Electric Power Corporation Metering Center, Chengdu, 610045, China \\ ${ }^{3}$ Huazhong University of Science and Technology, Wuhan, 430074, China \\ aemail: tangyi04@163.com
}

Keywords: Digital Electric Power Metering system; Quantization Error; IEC61850

\begin{abstract}
In digital electric power metering system, the sampled values of currents and voltages are transferred according to IEC61850-9-1/2 protocol. Quantization error will be introduced because the transferred data bit is limited. The quantization error introduced by IEC61850-9-1/2 is researched. The mathematical formula of uncertainty introduced by quantization error is achieved, and it accords well with the simulation results. The influence to actual meter of different accuracy levels is discussed
\end{abstract}

\section{Introduction}

Smart gird and smart substation are the technical trend of electric power system. The state grid corp. has already started the construction of smart grid and smart substations. In newly-built smart substations, the digital metering systems are employed. In this new metering system, secondary currents and voltages are transferred in the form of digital sampled values via the dedicated protocol of IEC61850-9-1/2. In contrast, the secondary currents and voltages are transferred in the forms of analog signals. Together with many advantages, the digital metering system brings about new technical issues, one of which is the quantization error introduced by IEC61850-9-1/2.

In smart or digital substations, the secondary currents and voltages output by measurement transformers are locally sampled, and the sampled values are assembled into data frames by merging unit (MU) according to IEC61850-9-1/2 protocol. The IEC61850-9-1/2 data frames will then be transferred to metering, relay protect and measurement devices. Figure 1 shows the structure mentioned above. Since the bits of sampled values in the data frames are limited, and thus the quantization error is inevitable.

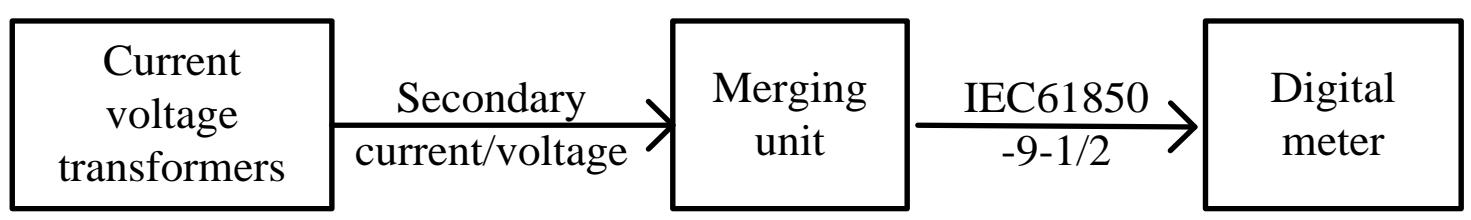

Figure 1 the structure of digital metering system.

The ascription of this quantization error is debatable. One viewpoint is that it belongs to electronic transformers [1], while the other is that it belongs to the MU [2]. However, the quantization is immanent and not ignorable in digital metering system [3]. Many literatures have been recently published regarding the metering algorithm [4], calibration[1][7] and traceability[6] solutions. There are few literatures researching the quantization error introduced by IEC61850-9-1/2 protocol. Literature [1] presents that the quantization error might be much bigger than calculation error under little current and voltages, and yet no detail quantitative analysis is presented. Literature [2] presents that the quantization error is small and IEC61850-9-1 and IEC61850-9-2 have quantization error of the same level. However, it has not researched the 
influence of different levels of primary current and voltages. In this paper, detail research on the quantization will be presented by means of principle analysis and numerical simulation, and the influence on the whole digital metering system will also be discussed.

\section{Definition of Quantization Error}

$x(t)$ is a continuous signal. Let discrete signal $x[k]$ be the sampled valued of $x(t)$. Then

$x[k]=x(k \Delta t), k=0,1,2, \ldots$

where, $\Delta t$ is the sampling time interval.

Let $x_{\mathrm{q}}[k]$ be the quantized sampled value. Then

$x_{q}[k]=x[k]+e[k]$

$e[k]$ is the quantization error of a single sampled value $x[k]$. $e[k]$ is an independent random variable with uniform distribution at $[-q / 2, q / 2]$. Fig.2 shows the distribution of probability density of quantization error. Hence, $q$ is the minimum quantization unit, meaning the quantity one bit stands by. Obviously, the mean value of $e[k]$ is 0 , the variance of $q$ is $q^{2} / 12$.

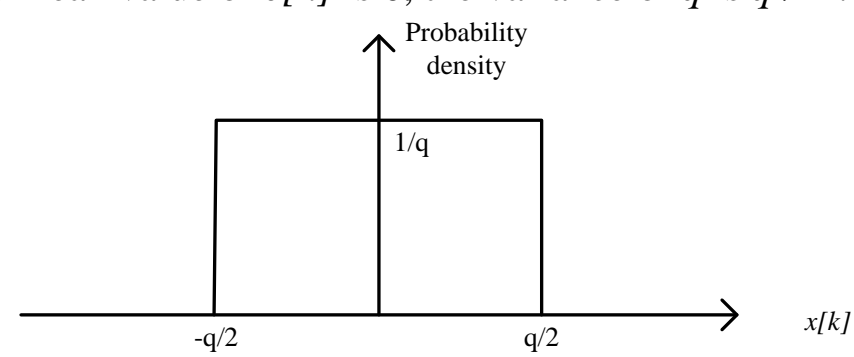

Fig.2 probability density of quantization error for uniform distribution

\section{Quantization Error in IEC61580-9-1/2}

According to IEC61850-9-1/2, the sampled values of secondary currents and voltages are packed in the data frames with limited bits. The quantized values can be expressed as below,

$$
\begin{aligned}
& u_{q}[\mathrm{k}]=\operatorname{round}\left(\frac{\sqrt{2} U \sin (\omega k \Delta t+\theta+\varphi)}{q_{u}}\right) \\
& i_{q}[\mathrm{k}]=\operatorname{round}\left(\frac{\sqrt{2} \operatorname{Isin}(\omega k \Delta t+\theta)}{q_{i}}\right)
\end{aligned}
$$

Where, $\mathrm{U}$ and I are the effective values of voltage and currents, $\theta$ is the sampling initial phase, $\varphi$ is the power angle, $\omega$ is the angular velocity, $q_{u}$ and $q_{i}$ are the minimum quantization units of voltage and current, $\Delta t$ is the sampling time interval, and round() is the rounding function , which makes the decimal be the nearest integer.

In IEC61850-9-1 [8], the transferred values $u_{q}[\mathrm{k}]$ and $i_{q}[\mathrm{k}]$ are the secondary voltages and currents, and it is stipulated that the rated voltage or current responds a fixed value of 0x2D41(11585). So, the quantization error has a constant proportion to the rated value. It can be expressed as below,

$$
\begin{aligned}
& q_{u}=\frac{u_{r}}{11585} \\
& q_{i}=\frac{i_{r}}{11585}
\end{aligned}
$$

where, $u_{r}$ and $i_{r}$ are the rated secondary voltage and current.

In IEC61850-9-2 [9], the transferred values are the primary voltages and currents, and technically the quantization units $q_{u}$ and $q_{i}$ can be arbitrarily configured. However, in order to enable the compatibility of different venders, IEC61850-9-2 LE, which is a limited edition and stipulates some configurations to be fixed values, is the widely-used protocol. In IEC61850-9-2 LE, $q_{u}$ and $q_{i}$ are constantly $0.01 \mathrm{~V}$ and $0.0001 \mathrm{~A}$, which means that 1 bit stands by $0.01 \mathrm{~V}$ and 0.0001A. We will use IEC61850-9-2 LE in following analysis. 


\section{Theoretical Analysis of Quantization Error}

In IEC61850-9 data frames, the transferred values can be expressed as below, $U_{q}[k]=U[k]+e_{u}[k]$

$I_{q}[k]=I[k]+e_{i}[k]$

Where, $U[\mathrm{k}]$ and $I[\mathrm{k}]$ are the real value before quantization, $e_{u}[\mathrm{k}]$ and $e_{i}[\mathrm{k}]$ are the quantization errors for a single sample, $\mathrm{U}_{\mathrm{q}}[\mathrm{k}]$ and $\mathrm{I}_{\mathrm{q}}[\mathrm{k}]$ are the quantized values. Then, the effective value (root-mean-square) of voltage, which is calculated with quantized values, is

$$
U_{q}^{2}=\frac{1}{N} \sum_{k=1}^{N}\left(U[k]+e_{u}[k]\right)^{2}
$$

Where, $N$ is the sample number that one period contains. $N$ is usually an even number according to protocol. Since $e_{u}[k]$ is a random variable, $U_{q}^{2}$ is also a random variable. Its variance can be expressed as below,

$$
D\left(U_{q}^{2}\right)=D\left(\frac{1}{N} \sum_{k=1}^{N}\left(U[k]+e_{u}[k]\right)^{2}\right)=\frac{1}{N} D\left(\sum_{k=1}^{N}\left(U[k]+e_{u}[k]\right)^{2}\right)
$$

We assume the $U[k]$ is ideal sinusoidal wave, and thus $\left(U[k]+e_{u}[k]\right)^{2}=\left(U[k+N / 2]+e_{u}[k+N / 2]\right)^{2}$, that is,

$\operatorname{cov}\left(\left(U[k]+e_{u}[k]\right)^{2},\left(U[k+N / 2]+e_{u}[k+N / 2]\right)^{2}\right)=\operatorname{cov}\left(\left(U[k]+e_{u}[k]\right)^{2},\left(U[k]+e_{u}[k]\right)^{2}\right)=D\left(\left(U[k]+e_{u}[k]\right)^{2}\right)$

Here, $\operatorname{cov}(X, Y)$ means the covariance of $X$ and $Y$. it can also got that,

$\operatorname{cov}\left(\left(U[\mathrm{i}]+e_{u}[\mathrm{i}]\right)^{2},\left(U[\mathrm{j}]+e_{u}[\mathrm{j}]\right)^{2}\right)=0 \quad(\mathrm{i}, \mathrm{j}=1,2, \ldots, \mathrm{N}, \mathrm{i} \neq \mathrm{j} \pm \mathrm{N} / 2)$

Then with the formula, $D(X, Y)=D(X)+D(Y)+2 \operatorname{cov}(X, Y)$, it can be got that,

$D\left(U_{q}^{2}\right)=D\left(\frac{1}{N} \sum_{k=1}^{N}\left(U[k]+e_{u}[k]\right)^{2}\right)=\frac{1}{N} \sum_{k=1}^{N} D\left(2\left\ulcorner\left(U[k]+e_{u}[k]\right)^{2}\right)\right.$

After some calculation, finally,

$D\left(U_{q}^{2}\right)=\frac{2 q_{u}^{2} U_{r m s}^{2}}{3 N}+\frac{q_{u}^{4}}{90 N U_{r m s}^{2}}$

Here, Urms is the root-mean-square of real voltage, U[k]. The normalized standard variance of Uq2, which is also the relative uncertainty, is

$\sigma\left(U_{q}{ }^{2}\right)_{\text {nom }}=\frac{\sqrt{D\left(U_{q}{ }^{2}\right)}}{\sqrt{U_{r m s}^{2}}}=\sqrt{\frac{2 q_{u}{ }^{2}}{3 N}+\frac{q_{u}^{4}}{90 N U_{r m s}^{2}}}$

Similarly, the relative uncertainty of Iq2 is,

$\sigma\left(\mathrm{I}_{q}^{2}\right)_{\text {nom }}=\sqrt{\frac{2 q_{i}^{2}}{3 N}+\frac{q_{i}^{4}}{90 N I_{\text {rms }}^{2}}}$

The calculated power with quantization error, Pq, can be calculated as below,

$P_{q}=\frac{1}{N} \sum_{k=1}^{N}\left(U[k]+e_{u}[k]\right)\left(I[k]+e_{i}[k]\right)$

We also take care of the covariance of $k$ th and $(k+N / 2)$ th adding elements, and after similar mathematical deduction, we can get the normalized standard variance (relative uncertainty) of $P_{q}$ $\sigma\left(\mathrm{P}_{q}\right)_{\text {nom }}=\frac{\sqrt{D\left(\mathrm{P}_{q}\right)}}{P}=\sqrt{\frac{q_{u}{ }^{2} q_{i}^{2}}{72 N U_{r m s}^{2} I_{r m s}^{2} \cos ^{2} \varphi}+\frac{q_{i}^{2}}{6 N I_{r m s}^{2} \cos ^{2} \varphi}+\frac{q_{u}^{2}}{6 N U_{r m s}^{2} \cos ^{2} \varphi}}$

Here, $\mathrm{P}$ is the power value. $P=U_{r m s} I_{r m s} \cos \varphi, \varphi$ is the power angle.

It can be seen according to (15), (16) and (18) that the uncertainty of effective value and power are related to many factors, including $q_{u}, q_{i}, N, U_{r m s}, I_{r m s}$ and $\cos \varphi$.

\section{Simulation of Uncertainty}

In order to verify the mathematical formula of uncertainty, we do some simulation with Matlab. According to actual usage of IEC61850-9-2LE, we set $u_{q}=0.01 \mathrm{~V}, i_{q}=0.001 \mathrm{~A}$ and $N=0.001 \mathrm{~A}$. $U_{r m s}, I_{r m s}$ and $\cos \varphi$ can be set unconstrainedly. By changing the sampling initial phase $\theta$, we get 
different quantization error $e_{u}[k]$ and $e_{i}[k]$. We did as many as 10000 times and then calculated the standard deviation. Table 1 shows the comparison of the theoretical and simulation result for square of effective voltage. It can be seen the difference is very small. Fig.3 shows the comparison of the theoretical and simulation result for power uncertainty. It can be seen that the theoretical and simulated results are very close, almost the same. Therefore, the validity of the theoretical analysis is verified.

Table 1 uncertainty of the square of effective voltage

\begin{tabular}{ccc}
\hline$U_{r m s}(\mathrm{kv})$ & Simulated uncertainty $\left(\times 10^{-7}\right)$ & theoretical uncertainty $\left(\times 10^{-7}\right)$ \\
\hline 500 & 0.0183 & 0.0183 \\
220 & 0.0411 & 0.0415 \\
110 & 0.0834 & 0.0830 \\
35 & 0.2590 & 0.2608 \\
10 & 0.9165 & 0.9129 \\
\hline
\end{tabular}

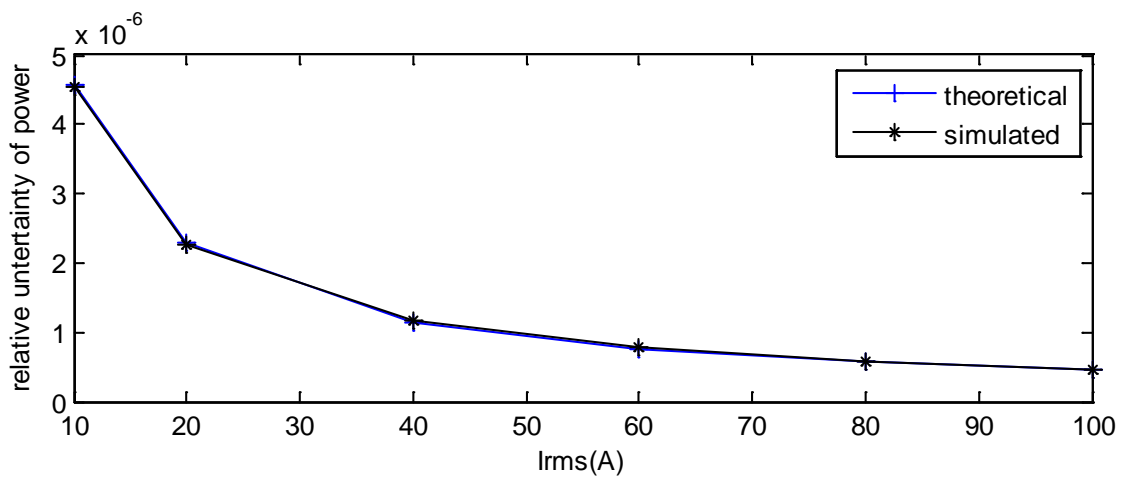

(a) $U_{r m s}=10 \mathrm{kV}, \cos \varphi=1, I_{r m s}$ varies

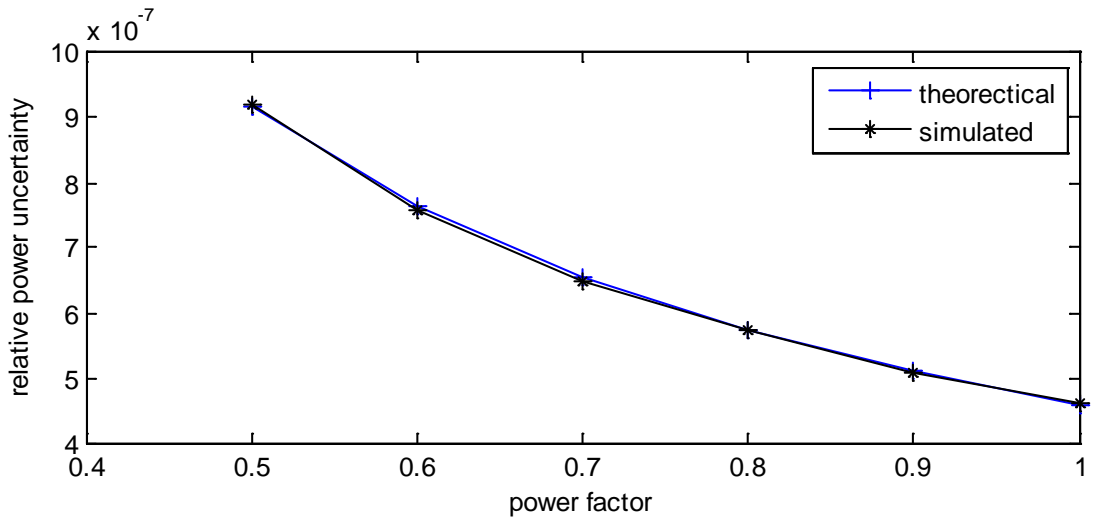

(b) $U_{r m s}=10 k V, U_{r m s}=100 A$, power factor $(\cos \varphi)$ varies

Fig.3 the comparison of simulated and theoretical power uncertainty

\section{Discussion for Quantization Error of IEC61850-9-1/2}

As mentioned above, in IEC61850-9-1, the quantization unit is has a constant proportion to rated current and voltage as (5) and (6). Taking (5) and (6) into (18), it can be got as below,

$$
\sigma\left(\mathrm{P}_{q}\right)^{\prime}{ }_{\text {nom }}=\sqrt{\frac{U_{r}^{2} I_{r}{ }^{2}}{11585^{4} \square 72 N U_{r m s}^{2} I_{r m s}^{2} \cos ^{2} \varphi}+\frac{I_{r}^{2}}{11585^{2} \square 6 N I_{r m s}^{2} \cos ^{2} \varphi}+\frac{U_{r}^{4}}{11585^{2} \square 6 N U_{r m s}^{2} \cos ^{2} \varphi}}
$$

It can be seen that the power uncertainty is only related to the values of $U_{r} / U_{r m s}$ and $I_{r} / I_{r m s}$. In practical usages, $U_{r} / U_{r m s}=1$, and $I_{r m s}$ can be smaller than $I_{r}$. According to related standards, the metering accuracy must be guaranteed when $I_{r m s}$ is as small as $0.01 I_{r}$. Table 2 shows the uncertainty introduced by IEC61850-9-1 quantization error when $\mathrm{I}_{\mathrm{rms}} / \mathrm{I}_{\mathrm{r}}$ varies. It can be seen that the uncertainty comes bigger when $I_{r m s} / I_{r}$ decreases. However, it can meet the requirement of 0.2S accuracy (installed meter's highest accuracy level, allowed error is $0.2 \%$ ) even when $\mathrm{I}_{\mathrm{rms}} / \mathrm{I}_{\mathrm{r}}$ is 0.01 . But, the uncertainty cannot 
meet the requirement of reference (standard) meter's accuracy levels such as 0.02S or 0.01S.

Table 2 uncertainty of power introduced by IEC61850-9-1 quantization error

\begin{tabular}{cccc}
\hline $\mathrm{I}_{\mathrm{rms}} / \mathrm{I}_{\mathrm{r}}$ & $\mathrm{U}_{\mathrm{rms}} / \mathrm{U}_{\mathrm{r}}$ & $\cos \varphi$ & Uncertainty (\%) \\
\hline 1 & 1 & 1 & 0.00056 \\
0.5 & 1 & 1 & 0.00088 \\
0.2 & 1 & 1 & 0.00201 \\
0.1 & 1 & 1 & 0.00396 \\
0.05 & 1 & 1 & 0.00789 \\
0.01 & 1 & 1 & 0.03940 \\
\hline
\end{tabular}

In the case of IEC61805-9-2, the quantization error introduced by protocol is related to primary current and voltage. Table 3 shows the uncertainties for different currents and voltages. It can be seen that when the currents and voltages are bigger than $5 \mathrm{~A}$ and $57.7 \mathrm{~V}$, the uncertainty is very small and can be ignored. When the current is as small as $1 \mathrm{~A}$ or even lower, the uncertainty cannot be ignored any more for $0.02 \mathrm{~S}$ or $0.2 \mathrm{~S}$ accuracy level. However, in actual substations, the primary voltage is usually bigger than $10 \mathrm{kV}$, and the primary current is usually bigger than $100 \mathrm{~A}$. So the quantization error is not big enough to influence the metering result. But in the case of lab testing, the primary and second current and voltage can be set arbitrarily. If we, in order to simplify the setting process, set the primary current and voltage as the same as secondary ones, the uncertainty as big as table 3 will be introduced and it will affect the testing results. Therefore, it is highly recommended that relative testing specification should stipulate the specific settings for primary currents and voltages.

Table 3 uncertainty of power introduced by IEC61850-9-2 quantization error

\begin{tabular}{cccc}
\hline $\mathrm{I}_{\mathrm{rms}}(\mathrm{A})$ & $\mathrm{U}_{\mathrm{rms}}(\mathrm{V})$ & $\cos \varphi$ & Uncertainty (\%) \\
\hline 600 & 220000 & 1 & $7.6101 \times 10^{-6}$ \\
600 & 10000 & 1 & $8.8715 \times 10^{-6}$ \\
600 & 57.7 & 1 & $7.7919 \times 10^{-4}$ \\
5 & 57.7 & 1 & $1.2079 \times 10^{-3}$ \\
5 & 57.7 & 0.5 & $2.4159 \times 10^{-3}$ \\
$1(20 \%$ of 5$)$ & 57.7 & 0.5 & $9.2648 \times 10^{-3}$ \\
$0.05(1 \%$ of 5$)$ & 57.7 & 0.5 & 0.18 \\
\hline
\end{tabular}

\section{Conclusion}

In digital electric power metering system, the currents and voltages are transferred according to IEC61850-9-1/2 protocol. It will introduce quantization error because of the limited data bit. Theoretical analysis and simulation shows the uncertainty introduced by quantization is related to quantization units, the sampling number in one period, the RMS of current and voltage and the power factor. For IEC61850-9-1, the influence of quantization error is only related to the proportion between actual and rated current/voltage. Its uncertainty can meet the requirement of installed meter (0.2S accuracy level) but it can affect reference (standard) meter (0.02S or 0.01S). For IEC61850-9-2 LE, the influence of quantization error is related to primary current and voltage. If the primary current and voltage are set as actual substation, the influence of quantization error can be ignored. But it can't be ignored if the primary current and voltage are set to extreme values in lab testing. It is highly recommended that relative testing specification should stipulate the specific settings for primary currents and voltages, when IEC61850-9-2 LE is used.

\section{Acknowledgement}

In this paper, the research was sponsored by the Technical Research Project of State Grid Sichuan Power Corporation (Project No. 52199713506L). 


\section{References}

[1] Li Qian, ZHANG Shu - han,LU Yi - biao,et al. The Study of On-site Metering Calibration of Fully Digital Power Metering System [J].Electrical Measurement \& Instrumentation, 2010, 47(538): 25-28

[2] Xie Dong, WU Tao, WU Bo - hua,et al. Discusion on Accuracy Class Issue for Digital Interface Electricity Meter [J]. Electrical Measurement \& Instrumentation, 2011, 48(548): 87-91 (In Chinese)

[3] SUN Wei-ming,LIN Guo-ying. Research of Calibaration Method for Electrical Energy Meter Based on IEC61850 [J].Electrical Measurement \& Instrumentation, 2010, 47(7A): 13-15. (In Chinese)

[4] WANG Le - ren ,LEI Min, ZHANG Shu-han . Research on Electric Algorithm by IEC 61850-9-1 Protocol [J]. Electrical Measurement \& Instrumentation, 2011, 48(548): 87-91 (In Chinese)

[5] WAN Bo, SU Rui . Implementation of Sampled Value Tranmission Complying With IEC 61850-9-2 [J]. Power System Technology, 2011, 48(548): 87-91 (In Chinese)

[6] CHEN Rui-min, LI Xin,KONG Zhen-min,ea al.Meter Calibration Technology for Digital Watt-hour Meters Based on Value Traceability. [J]. Electrical Measurement \& Instrumentation, 2012, 49(562): 38-41 (In Chinese)

[7] SUN Wei-ming, LIN Guo-ying, ZHOU Shang-li, et al. Total System Accuracy of Metering Devices in Digital Substation and Its Long Term Operating Characteristics[J]. Electrical Measurement \& Instrumentation, 2010, 47(8): 27-31. (In Chinese)

[8] IEC61850 IEC. 9-1 Communication networks and systems in substations, part 9-1: Specific Communication Service Mapping (SCSM)-sampled values over multi-drop point link, 2003.

[9] IEC61850 IEC. 9-2 Communication networks and systems in substations, part 9-2: Specific Communication Service Mapping (SCSM)-sampled values over ISO[J]. IEC8802-3, 2004. 